\title{
Efecto de la adición de subproductos agroindustriales en las propiedades físicas de un bioplástico almidón-gelatina
}

\author{
Effect of agro-industrial by-products addition on the physical properties of a starch-gelatin bioplastic
}

\begin{abstract}
Blanca Aurora Francisco Ponce', Isamar Maydeth Vidal Silva', Yanik Ixchel Maldonado Astudillo², Javier Jiménez Hernández², Verónica Flores Casamayor², Gerónimo Arámbula Villa², Ricardo Salazar ${ }^{4 *}$

Facultad de Ciencias Químico-Biológicas. Universidad Autónoma de Guerrero. Av. Lázaro Cárdenas s/n. Ciudad Universitaria Sur, Col. La Haciendita, 39090. Chilpancingo de los Bravo, Guerrero, México.

2 Centro de Innovación, Competitividad y Sostenibilidad (CICS). Universidad Autónoma de Guerrero. Calle Pino S/N Col. El Roble, 39640. Acapulco, Guerrero, México.

Centro de Investigación y de Estudios Avanzados del Instituto Politécnico Nacional (CINVESTAV IPN), Unidad Querétaro, Libramiento Norponiente \# 2000, Fraccionamiento Real de Juriquilla, 76230. Querétaro, Querétaro, México.

4 CONACyT-Universidad Autónoma de Guerrero, Av. Javier Méndez Aponte No. 1, Fracc. Servidor Agrario, 39070. Chilpancingo de los Bravo, Guerrero, México.
\end{abstract}

\section{RESUMEN}

En este trabajo se reporta el efecto de la adición de subproductos de mango, jamaica y café a una formulación de almidón-gelatina para elaborar un bioplástico en forma de cuchara. Se encontró que la adición de los subproductos mejoró las propiedades mecánicas del bioplástico debido a la compatibilidad de sus componentes observados a nivel microscópico (la dureza aumentó de 190 hasta 290 N). Asimismo, las propiedades físicas de los bioplásticos como el ángulo matiz (63-89), y los índices de solubilidad y absorción en agua se modificaron por la adición de subproductos. Se observó que las condiciones de máxima estabilidad de los bioplásticos obtenidas a partir del modelado de las isotermas de adsorción a $35^{\circ} \mathrm{C}$ con la ecuación de GAB y el cálculo el volumen de microporo correspondieron a una actividad de agua cercana a 0.40 , independientemente del subproducto agregado. Las distintas formulaciones exhibieron un evento endotérmico a una temperatura de $180^{\circ} \mathrm{C}$, asociado a un proceso de fusión, así como una elevada tasa de degradación en suelo después de $120 \mathrm{~h}$. Los subproductos probaron ser un refuerzo en la formulación de bioplásticos. El uso de subproductos de mango, café y jamaica representan una alternativa interesante para incrementar su potencial tecnológico.

Palabras clave: Subproducto; Bioplástico; Características físicas.

\section{ABSTRACT}

This study reports the effect of mango, jamaica and coffee by-products addition into formulation of starchgelatin for elaborating a spoon-shaped bioplastic. Such additions improved the bioplastics mechanical properties due to the compatibility of their components, observed at microscopic level (hardness was increased in a range of 190 to $290 \mathrm{~N}$ ). In the same way, the bioplastics physical properties such as the hue angle values (63-89), solubility and absorption index, were modified with the by-product's addition. To obtain the spoon-shaped bioplastics conditions of maximum stability $\left(a_{w} \approx 0.4\right)$ at $35^{\circ} \mathrm{C}$, we employ the GAB equation and the moisture content corresponding to micropore volume. The different formulations exhibited an endothermic event at $180^{\circ} \mathrm{C}$, associated with a melting process, as well as a high degradation rate in soil after $120 \mathrm{~h}$. The by-products proved to be a reinforcement in the bioplastics formulation. The use of by-products of mango, coffee and jamaica represent an interesting alternative to increase their technological potential. Keywords: By-product, Biopolymer, Physical properties

\section{INTRODUCCIÓN}

En la actualidad los plásticos convencionales a base de petróleo constituyen una grave amenaza ambiental en todo el mundo. Esto se debe al hecho de que proceden de combustibles fósiles no renovables y su acumulación en suelos y mares ocasiona altos niveles de contaminación porque no pueden ser fácilmente degradados por el entorno. Actualmente se estima que aproximadamente 8 millones de toneladas de plásticos desechados terminan en el océano cada año (Krishnamurthy y Amritkumar, 2019) y se espera que esta cifra continúe aumentando debido al uso generalizado de productos de origen petroquímico como el tereftalato de polietileno (PET), poliestireno (PS), polietileno (PE) y polipropileno (PP). Por esta razón, la creciente preocupación por la contaminación ambiental originada por los plásticos convencionales ha inspirado el desarrollo de plásticos biodegradables derivados de fuentes renovables como son los polímeros naturales (ácido poliláctico, polihidroxibutiratos, polihidroxialcanoatos, almidón, quitosano, entre otros) (Brizga et al., 2020). La biodegradabilidad se define como la descomposición natural y no contaminante de un producto por la acción de agentes biológicos (Imam et al., 2000).

El almidón es el polímero natural más utilizado en la fabricación de plásticos biodegradables por su renovabilidad, disponibilidad y bajo costo (Lubis et al., 2018), debido a que 
se encuentra ampliamente distribuido en la naturaleza al ser el principal polisacárido de reserva energética en plantas; no obstante, su uso individual proporciona bioplásticos con propiedades insatisfactorias en aplicaciones comerciales como el servicio, empaque o envasado de alimentos y bebidas. Entre las principales desventajas asociadas a los bioplásticos de almidón, en comparación con los plásticos convencionales actualmente en uso, se citan una limitada estabilidad dimensional cuando son expuestos a la humedad; descomposición térmica a bajas temperaturas (Avérous y Boquillon, 2004; Yang et al., 2019); y propiedades mecánicas inferiores como una menor resistencia a la tracción y alargamiento (Krishnamurthy y Amritkumar, 2019).

Un enfoque para mejorar las características de los bioplásticos de almidón es la adición como refuerzo de fibras vegetales como la celulosa, hemicelulosa y lignina, principalmente debido a su amplia disponibilidad, biodegradabilidad, y atractivas propiedades mecánicas (ductilidad y elevada resistencia a la fractura). Diversos estudios recientes han demostrado que la adición de fibras lignocelulósicas incrementa la resistencia de bioplásticos elaborados a partir de almidón (Yang et al., 2019).

Por otra parte, en los últimos años ha adquirido especial importancia el uso de residuos de agricultura en forma de semillas, cáscara y bagazo como fuente potencial de fibra debido a que se trata de materiales abundantes, generalizados y fácilmente disponibles. La utilización de residuos agrícolas además proporciona ventajas importantes como la reducción de los costos y una menor necesidad de la gestión tradicional de los desechos, favoreciendo la economía, el medio ambiente y la tecnología a largo plazo (Ashori y Nourbakhsh, 2010; Väisänen et al., 2016).

Diversos autores han reportado el uso de subproductos agrícolas en matrices poliméricas, como la adición de bagazo de caña, de agave, fique, yute, entre otros (Velásquez et al., 2016; Aranda et al., 2015) donde se ha observado una mejora de sus propiedades mecánicas, térmicas, así como una mayor compatibilidad de sus componentes a nivel microscópico (la hidrofilicidad y el débil entrecruzamiento de las fibras incrementa la adhesión interfacial de los componentes dentro de la matriz polimérica). Williamson y Hatzakis (2019), demostraron que los lípidos extraídos de los residuos del café son ingredientes promisorios en la elaboración de bioplásticos. En el mismo sentido, Moro et al. (2019), reportaron que la adición de cáscara de maracuyá mejoró las propiedades mecánicas de un bioplástico preparado a partir de almidón extrudido. Sin embargo, a pesar de los estudios exitosos reportado en la literatura, todavía se requieren estudios para la revalorización de subproductos agroindustriales como agentes de refuerzo para la mejora de las características de bioplásticos elaborados a base de almidón. Se sabe que las fibras de cáscara de mango, cáliz de jamaica y residuos de café tostado son ricos en fibras como celulosa, hemicelulosa y lignina, así mismo estos no poseen valor económico (McNutt, 2019; Mayo-Mayo et al., 2020) por lo que representan un recurso sostenible para la producción de bioplásticos.
Por lo tanto, el objetivo del presente estudio fue evaluar el efecto de la adición de subproductos de mango, jamaica y café en las propiedades físicas (color, textura, morfología, actividad de agua, adsorción de humedad, transiciones térmicas, absorción y solubilidad en agua) de un bioplástico de almidón de maíz y gelatina utilizado para obtener cucharas biodegradables.

\section{MATERIALES Y MÉTODOS}

El tipo de estudio fue experimental, se utilizó cáscara de mango (Mangifera indica), cáliz de jamaica cocido (Hibiscus sabdariffa) y residuos de café tostado (Coffea arabica) que se colectaron en el municipio de Chilpancingo del estado de Guerrero, México. La recolección en bolsas de plástico de los residuos de cáscara de mango criollo y cálices cocidos de jamaica se realizó en un mercado local, y los residuos de café tostado se recolectaron en una cafetería local. Se utilizó almidón ceroso de maíz suministrado por Ingredion $\circledast$. El ácido clorhídrico $(\mathrm{HCl})$, el hidróxido de sodio $(\mathrm{NaOH})$, el glicerol y las sales usadas para la elaboración de las isotermas de adsorción se adquirieron en Sigma-Aldrich (St. Louis, MO, EU) o Golden Bell reactives (Zapopan, Jalisco, México) y fueron grado analítico.

\section{Procesamiento de los subproductos}

Los residuos de café tostado se secaron en un horno de convección forzada y capacidad de $30 \mathrm{~L}$ (Luzeren modelo DHG-9070A, Beijing, China) a $60^{\circ} \mathrm{C}$ durante 24 horas. La cáscara de mango y el cáliz cocido de jamaica se sometieron a inactivación enzimática mediante ebullición en agua durante $5 \mathrm{~min}$, posteriormente se colocaron en un horno de convección forzada a $60^{\circ} \mathrm{C}$ durante 48 horas. Por último, los residuos fueron pulverizados en un molino de café (KRUPS modelo GX410011, Solingen, Germany), tamizados en una malla con una apertura de $425 \mu \mathrm{m}$ y almacenados en frascos herméticamente cerrados y cubiertos de la luz a temperatura ambiente $\left(\approx 25^{\circ} \mathrm{C}\right.$ ) (Mayo-Mayo et al., 2020).

\section{Hidrólisis de almidón}

Se preparó una solución al 30\% en peso de almidón, adicionando $4 \%$ de $\mathrm{HCl}$ y el resto de agua destilada. La solución se agitó de forma constante a una velocidad de 500 rpm en un agitador mezclador de propela 20L (OS20-S, DLAB Scientific Co., Ltd., China) a $40^{\circ} \mathrm{C}$ durante 3 horas. Se ajustó el $\mathrm{pH}$ a 5.5 adicionando $\mathrm{NaOH}$, utilizando un potenciómetro (Starter-3100, Ohaus, NJ, EE. UU) y se refrigeró durante 24 horas. La mezcla se repartió en tubos cónicos Falcon de 50 $\mathrm{mL}$ y se centrifugaron (centrífuga Eppendorf modelo 5810R, Hamburgo, Alemania) a $1800 \times$ g por 5 min. Posteriormente, se descartó el sobrenadante para recuperar el sedimento, y resuspender en agua. El proceso anterior se llevó a cabo cuatro veces. El almidón resultante se secó durante 24 horas en un horno de convección forzada, se pulverizó y almacenó a temperatura ambiente (García-Gurrola et al., 2019). 


\section{Elaboración del biopolímero}

Se estableció la formulación de los bioplásticos a partir de almidón de maíz hidrolizado de acuerdo con Rodríguez et al. (2014). Brevemente, se realizó una solución 1:1 de almidón hidrolizado y gelatina ( $15 \mathrm{~g}$ de cada uno), adicionando $50 \mathrm{~mL}$ de agua destilada y $0.2 \mathrm{~g}$ de glicerol (plastificante) como tratamiento control. Se realizaron 3 tratamientos adicionando 2 g de cada subproducto. La mezcla se calentó a $60^{\circ} \mathrm{C}$, se vació en moldes de silicón y finalmente se secó por 24 horas a $40^{\circ} \mathrm{C}$, obteniéndose los bioplásticos. Las muestras elaboradas y su codificación fueron: tratamiento control de almidón de maíz (C), adición de cáscara de mango (CM), adición de cáliz de jamaica cocido (CJ), adición de residuos de café tostado (CC).

\section{Análisis Físicos}

El color se analizó mediante un colorímetro portátil (X-Espectrofotómetro Rite Modelo Ci62, X-Rite Incorporated, Grandville, MI, EE. UU.). El ángulo de Hue $\left(\mathrm{H}^{*}\right)$ se calculó a partir de los valores determinados de CIE $L^{*}, a^{*} y^{*}: H^{\circ}=\tan ^{-1}$ $\left(b^{*} / a^{*}\right)$, donde $a^{*}=$ [negativo (verde) a positivo (rojo)] y b* [negativo (azul) a positivo (amarillo)]. El iluminante empleado fue D65 y la posición estándar del observador fue $10^{\circ}$. La fuerza de fractura (FF) se determinó con un analizador de textura (Stable Micro Systems modelo TA-XT plus, Godalming, Reino Unido) con una sonda tipo punzón (ángulo de punta de $22^{\circ}$ ) a una velocidad de ensayo $2 \mathrm{~mm} \mathrm{~s}^{-1}$. La actividad de agua $\left(a_{w}\right)$ se evaluó a $25^{\circ} \mathrm{C}$ con un medidor de actividad de agua de punto de rocío Aqualab ${ }^{\circledR}$ 4te (Decagon Devices, Inc., Pullman, WA, EE. UU.).

\section{Índice de solubilidad en agua (ISA) e índice de absorción de agua (IAA)}

La capacidad de absorción de agua de los bioplásticos se midió de acuerdo con el método estándar ASTM (2005). Se dispersó $2.5 \mathrm{~g}$ de muestra triturada de cada tratamiento en $40 \mathrm{~mL}$ de agua destilada en un tubo cónico y se colocó en baño maría a $40^{\circ} \mathrm{C}$ durante 1 hora. El tubo se centrifugó a $1800 \times$ g durante 20 minutos a una temperatura de $27^{\circ} \mathrm{C}$. El gel sedimentado se separó por decantación y lo floculado se drenó en tubos de metal y se evaporó durante 24 horas a $80^{\circ} \mathrm{C}$. El Índice de solubilidad en agua (ISA) e Índice de absorción de agua (IAA) se calcularon de la siguiente manera:

$$
\begin{gathered}
I S A=\frac{\text { Peso del residuo de evaporación }(g)}{\text { Peso seco de la muestra }(g)} \times 100 \\
I A A=\frac{\text { Peso del residuo seco }(g)}{\text { Peso de la muestra inicial }(g)} \times 100
\end{gathered}
$$

\section{Morfología}

Se utilizó un Microscopio Electrónico de Escaneo Ambiental (ESEM, modelo Philips XL30 con un haz de 3 kV y un detector gaseoso de electrones secundarios. Las imágenes se tomaron a 500X y 1 Torr. Las muestras se recubrieron con grafito utilizando un pulverizador JFC-1100 (JEOL, Akishima, Japón).

\section{Isotermas de adsorción}

Se realizaron por duplicado a $35{ }^{\circ} \mathrm{C}$. Los datos de adsorción de humedad se obtuvieron por el Método Gravimétrico. Las muestras se colocaron en celdas de equilibrio que contenían soluciones saturadas que proporcionaron un rango de $\mathrm{a}_{\mathrm{w}}$ de 0.108 a 0.821 ; se determinó la variación en el peso que denotaba la adsorción de humedad hasta llegar al equilibrio. El modelo Guggenheim-Anderson-De Boer (GAB) se utilizó para ajustar los datos experimentales de las isotermas de adsorción (Al-Muhtaseb et al., 2004) de los bioplásticos:

$$
M=\frac{M_{0} C K a_{w}}{\left(1-K a_{w}\right)\left(1-K a_{w}+C K a_{w}\right)}
$$

Donde: $M\left(\mathrm{~g} \mathrm{H}_{2} \mathrm{O} / 100 \mathrm{~g}\right.$ s.s.) el contenido de humedad en equilibrio, $a_{w}$ es la actividad de agua, $M_{o}$ ( $\mathrm{g} \mathrm{H}_{2} \mathrm{O} / 100 \mathrm{~g}$ s.s.) es el contenido de humedad teórico de la monocapa, $\mathrm{C}$ y $\mathrm{K}$ son constantes.

Para la determinación del contenido de humedad correspondiente al volumen de microporo (n0) se utilizó la ecuación de Dubinin-Radushkevich (DR):

$$
\log n=\log n_{o}-B \log ^{2}\left(\frac{P v^{o}}{P v}\right)
$$

Donde: $n$ ( $\mathrm{g} \mathrm{H}_{2} \mathrm{O} / 100 \mathrm{~g}$ s.s.) el contenido de humedad en equilibrio, $n_{0}\left(\mathrm{~g} \mathrm{H}_{2} \mathrm{O} / 100 \mathrm{~g}\right.$ sólido seco) el volumen total de microporos accesibles al adsorbato, $B$ una constante relativa a la estructura microporosa del adsorbente, $P v^{\circ}$ es la presión de vapor del agua pura y $P v$ la presión de vapor del agua en el adsorbente.

\section{Calorimetría diferencial de barrido (DSC)}

Se utilizó un equipo DSC (Mettler-Toledo ${ }^{\circ}$ ), modelo 821e, Suiza) controlado por el software STARe versión 10.0. Se pesó $6 \mathrm{mg}$ de muestra en un crisol de aluminio y se sometió a una rampa de calentamiento con un rango de temperatura de $30-350^{\circ} \mathrm{C}$ y una velocidad de calentamiento de $10^{\circ} \mathrm{C} / \mathrm{min}$.

\section{Prueba de degradación en suelo}

Los biopolímeros fueron sumergidos en suelo a una $\mathrm{HR}=100 \%$ durante 5 días, donde se analizaron visualmente a las 0, 48 y 120 horas (López-Velázquez et al., 2019).

\section{Análisis estadístico}

Los datos fueron analizados como medias y desviación estándar (Media $\pm D S$ ). Se utilizó un análisis de varianza ANOVA de una vía. La comparación de medias se realizó con la prueba de Tukey $(p<0.05)$ utilizando el software Statistical Package for the Social Sciences (SPSS Statistics 25.0).

\section{RESULTADOS Y DISCUSIÓN}

\section{Caracterización del biopolímero}

Se desarrollaron y caracterizaron bioplásticos de almidón, gelatina y glicerol en forma de cucharas adicionadas con cáscara de mango (CM), residuos de café tostado (CC) y cálices de jamaica cocidos (CJ) (Figura 1). 


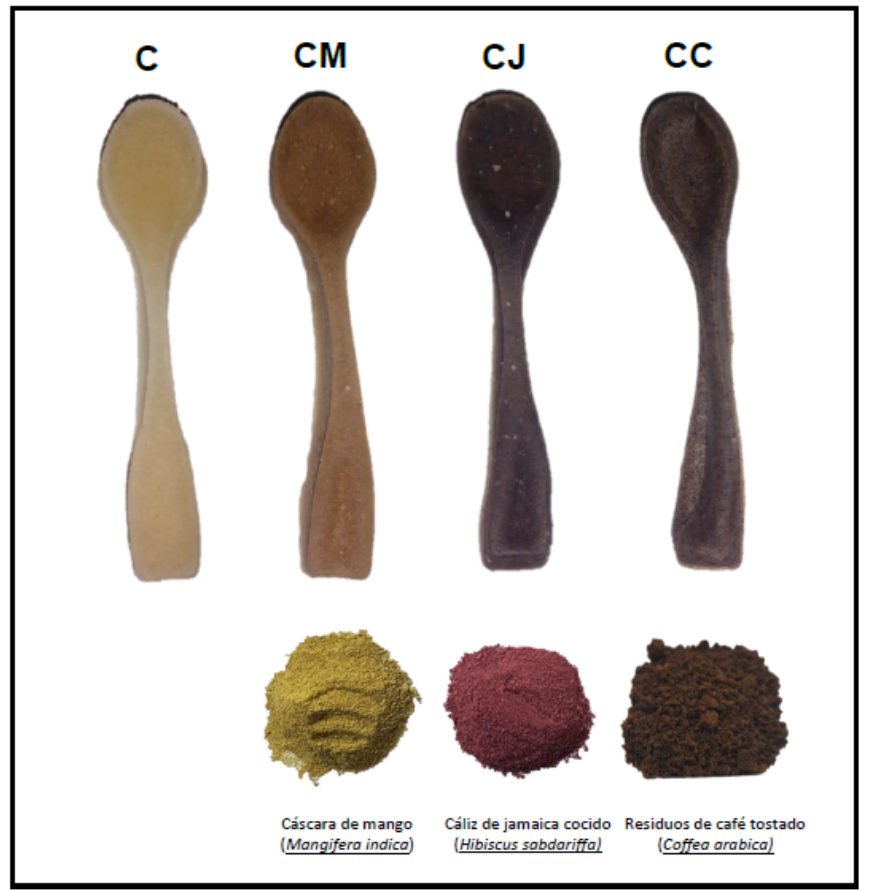

Figura 1. Bioplásticos obtenidos por la adición de subproductos de mango, jamaica y café. Control (C), cáscara de mango (CM), cálices de jamaica cocidos (CJ), residuos de café tostado (CC)

Figure 1. Bioplastics obtained by the addition of mango, roselle and coffee by-products. Control (C) mango peel (CM), decocted roselle calyces (CJ), spent coffee grounds (CC)

Los resultados de las pruebas de color y textura se muestran en la Tabla 1. Dado que el color es un factor importante en términos de apariencia general y aceptación por parte del consumidor, es necesario informar esta propiedad en los biopolímeros elaborados. Se observó que $C$ exhibió el mayor valor de luminosidad $\left(L^{*}=58\right)$ y el color más saturado $\left(C^{*}=18.8\right)$, con un tono amarillo confirmado por el ángulo $\mathrm{H}^{\circ}=89.24$, que se explica por la incorporación de la grenetina a la formulación, que posee un color amarillo característico. Como se esperaba, hubo un cambio de tonalidad, así como una disminución significativa en la luminosidad y cromaticidad de las cucharas en $C M\left(L^{*}=28.61, C^{*}=14.42\right), C J\left(L^{*}=21.41\right.$, $\left.C^{*}=4.63\right)$ y $C C\left(L^{*}=28.84, C^{*}=5.41\right)$, por efecto de la adición de los residuos, que es consistente con la coloración propia de los mismos. Los residuos de mango, jamaica y café mostraron una coloración café claro, rojo y café oscuro respectivamente. Esta disminución también se favoreció por reacciones de oscurecimiento no enzimático y la degradación de algunos pigmentos como los carotenoides y las antocianinas presentes en los subproductos por las condiciones de $\mathrm{pH}$ y temperatura que tienen lugar durante el procesamiento. Resultados similares han sido reportados por Santillán-Moreno et al. (2011), quienes registraron cambios en las tonalidades de mezclas extruidas de almidón de maíz y proteína de suero de leche debido a la adición de fibra de agave (valores mayores de luminosidad en las mezclas con bajo contenido de fibra) debido a la coloración propia de los ingredientes y al oscurecimiento por cambios químicos ocurridos durante el procesamiento.

El análisis de la fuerza de fractura (FF) se utiliza para estimar la resistencia y dureza de matrices poliméricas. El menor valor de FF se observó en el bioplástico C $(94.03$ $\mathrm{N})$, mientras que $\mathrm{CM}, \mathrm{CJ}$ y CC mostraron un aumento de la dureza, en donde CM presentó la mayor FF (290.15 N), por lo que la adición de los subproductos de mango incrementó la resistencia de esta cuchara en $200 \%$. Este comportamiento coincide con resultados reportados en la literatura sobre bioplásticos de almidón reforzados con diferentes tipos de fibras. Jumaidin et al. (2020) observaron una mejora significativa de la resistencia y el módulo de tracción en biopolímeros de almidón tras la incorporación de fibra de sisca (Imperata cylindrica). Syamani et al. (2020) observaron que la incorporación de fibra de celulosa aumentó la resistencia a la tracción hasta un $184 \%$ en un bioplástico a base de almidón de yuca, considerándose adecuado para competir en resistencia con el polímero sintético de polietileno de baja densidad. Este aumento en la rigidez de la matriz polimérica se ha atribuido a la compatibilidad del almidón con la fibra de los subproductos, debido a que la estructura química de ambos compuestos es relativamente polar y conduce a la formación de enlaces de hidrógeno entre los numerosos grupos hidroxilos (Wang et al., 2018). La mejora en la resistencia del biopolímero varía dependiendo del origen, concentración y longitud de la fibra (Avérous y Boquillon, 2004; Gáspar et al., 2005).

En la Tabla 2 se reportan los valores de IAA e ISA de los distintos bioplásticos elaborados. Cuando las moléculas de agua atacan los puentes de hidrógeno de los materiales los debilitan y disminuyen las propiedades funcionales de

Tabla 1. Evaluación de las características físicas de los bioplásticos. Table 1. Evaluation of bioplastics physical characteristics.

\begin{tabular}{lcccc}
\hline Tratamiento & L* & C $^{*}$ & Ho $^{*}$ & FF (N) \\
\hline C & $58.08 \pm 1.6 \mathrm{a}$ & $18.80 \pm 0.4 \mathrm{a}$ & $89.24 \pm 0.9 \mathrm{a}$ & $94.03 \pm 16.5 \mathrm{~d}$ \\
CM & $28.61 \pm 1.8 \mathrm{~b}$ & $14.42 \pm 0.2 \mathrm{~b}$ & $70.71 \pm 0.6 \mathrm{~b}$ & $290.15 \pm 32.9 \mathrm{a}$ \\
CJ & $21.41 \pm 1.6 \mathrm{c}$ & $4.63 \pm 1.4 \mathrm{c}$ & $54.52 \pm 2.3 \mathrm{~d}$ & $249.40 \pm 23.5 \mathrm{~b}$ \\
CC & $28.84 \pm 0.4 \mathrm{~b}$ & $5.41 \pm 1.6 \mathrm{c}$ & $66.11 \pm 2.8 \mathrm{c}$ & $190.22 \pm 37.1 \mathrm{c}$ \\
\hline
\end{tabular}

Luminosidad $\left(\mathrm{L}^{*}\right)$, Croma $\left(\mathrm{C}^{*}\right)$, Ángulo Matiz $\left(\mathrm{H}^{\circ}\right)$, Fuerza de fractura $(\mathrm{FF})$, Control $(\mathrm{C})$, cáscara de mango (CM), cálices de jamaica cocidos (CJ), residuos de café tostado (CC). Letras distintas en la misma columna indican diferencias significativas $(p<0.05)$ 
Tabla 2. Determinación del Índice de Solubilidad en Agua (ISA) e Índice de Absorción en Agua (IAA) y actividad de agua de los bioplásticos.

Table 2. Determination of bioplastics Water Solubility Index (ISA), Water Absorption Index (IAA) and water activity.

\begin{tabular}{lccc}
\hline Tratamiento & ISA (g sólido/100 g muestra) & IAA (g gel/100 g muestra) & Actividad de agua $\left(\mathrm{a}_{\mathrm{w}}\right.$ ) \\
\hline $\mathrm{C}$ & $38.52 \pm 0.7 \mathrm{~b}$ & $4.61 \pm 0.3 \mathrm{a}$ & $0.34 \pm 0.01 \mathrm{a}$ \\
$\mathrm{CM}$ & $40.63 \pm 0.8 \mathrm{a}$ & $2.18 \pm 0.1 \mathrm{~b}$ & $0.26 \pm 0.04 \mathrm{C}$ \\
$\mathrm{CJ}$ & $40.77 \pm 0.1 \mathrm{a}$ & $2.53 \pm 0.1 \mathrm{~b}$ & $0.27 \pm 0.02 \mathrm{C}$ \\
$\mathrm{CC}$ & $36.26 \pm 0.8 \mathrm{c}$ & $2.69 \pm 0.1 \mathrm{~b}$ & $0.31 \pm 0.01 \mathrm{~b}$ \\
\hline
\end{tabular}

Control (C), cáscara de mango (CM), cálices de jamaica cocidos (CJ), residuos de café tostado (CC). Letras distintas en la misma columna indican diferencias significativas $(p<0.05)$

los mismos (Kaisangsri et al., 2014). El bioplástico C mostró el mayor valor de IAA, en contraste CM exhibió el menor. Se puede apreciar que la adición de subproductos redujo significativamente los valores de IAA de los bioplásticos. Este comportamiento puede ser atribuido al incremento en el contenido de fibra insoluble presente en los subproductos que limita la absorción de agua en los bioplásticos (Abarca, 2010).

El ISA está relacionado con la presencia de enlaces covalentes o interacciones que reducen la solubilidad en agua de los bioplásticos (Kaisangsri et al., 2014). En este sentido, los bioplásticos CM y CJ mostraron los valores del índice de solubilidad mayores. Estos resultados pueden ser explicados con base en la presencia de fibra y compuestos bioactivos solubles presentes en la cáscara de mango y en los cálices de jamaica cocidos (Mayo-Mayo et al., 2020).

Se evaluó la $a_{w}$ de los bioplásticos, definida como una medida de la reactividad del agua para llevar a cabo reacciones de deterioro, se considera factor importante para determinar la vida útil de diversos productos, ya que establece el límite para el desarrollo de microorganismos. Se ha reportado que los microorganismos sólo pueden proliferar en ambientes donde la $a_{w}$ es mayor a 0.60 debido a la disponibilidad de agua que poseen para realizar procesos metabólicos (Lucas et al., 2008). Todas las formulaciones presentan una $a_{w}$ inferior $(<0.4)$, por lo que el riesgo de contaminación por proliferación de microorganismos disminuye en los bioplásticos.

La morfología de los biopolímeros se caracterizó para observar la compatibilidad entre los componentes de los bioplásticos (Figura 2). Se observó que la superficie presentaba diferencias por efecto de la adición de los subproductos. En la micrografía de C (Figura 2A) la morfología es más lisa y homogénea en comparación con las otras formulaciones. La estructura compacta observada del polímero de almidón es indicador de una mayor integridad estructural (Mali et al., 2002).

Las micrografías (Figura 2B-D) de CM, CJ y CC revelaron en cambio, una morfología más heterogénea en comparación con C, observándose la formación de múltiples poros desiguales en la matriz polimérica, mientras que otras regiones eran más compactas y con menor rugosidad que la superficie de la cuchara C. La presencia de estas regiones con una dispersión más uniforme indicaba una fuerte adhesión

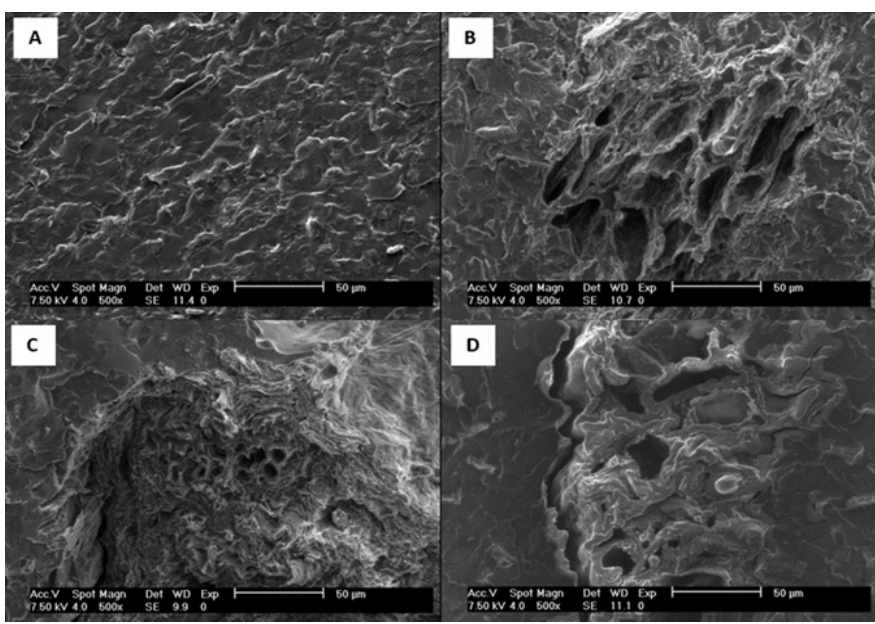

Figura 2. Microscopía electrónica de barrido. Superficie de los bioplásticos en forma de cuchara. A) C; B) CM; C) CJ; D) CC.

Figure 2. SEM micrographs. Surface morphology of spoon-shaped bioplastics. A) C; B) CM; C) CJ; D) CC.

entre el almidón y la fibra de los residuos agroindustriales. Este comportamiento es característico de los compuestos amiláceos biodegradables reforzados con fibras lignocelulósicas (Curvelo et al., 2001). Torres et al. (2019) examinaron la morfología de un bioplástico desarrollado a base de almidón de papa y reforzado con cáscaras de castaña, y observaron una distribución homogénea del residuo agroindustrial lignocelulósico en la matriz polimérica, lo que sugería una adecuada interacción de los componentes.

Una buena adherencia entre el material de refuerzo y la matriz conduce a una interfaz resistente, provocando un aumento de su rendimiento mecánico (De Carvalho et al., 2018), como permitieron comprobar los resultados del análisis de FF, donde la adición de los subproductos incrementó al doble la resistencia de las cucharas.

Por otra parte, la presencia de poros en la superficie de las cucharas se puede atribuir a la formación de agregados por efecto de la concentración de fibra en la formulación de las cucharas. Esto fue demostrado por De Carvalho et al. (2018), quienes observaron que la adición de $0.5 \%$ y $1 \%$ de fibra de bagazo de yuca a un biopolímero de almidón generó una matriz continua y cohesiva, pero un aumento de la concentración de material fibroso en $1.5 \%$ redujo la homogeneidad de la superficie generando poros y grietas en 
la matriz polimérica por la formación de agregados de fibra. Por lo tanto, esto sugiere que es posible modificar la cantidad de fibra adicionada a la matriz de almidón con la finalidad de mejorar la interacción entre ambos compontes y obtener cucharas más compactas.

\section{Establecimiento de las condiciones óptimas de almace- namiento}

Las isotermas de sorción relacionan la $\mathrm{a}_{\mathrm{w}} \mathrm{y}$ humedad en equilibrio a una temperatura constante, permitiendo determinar su comportamiento bajo condiciones específicas. Esto es importante para conocer las condiciones óptimas de almacenamiento, secado y predecir la vida útil de un producto (Navia et al., 2011). La Figura 3 muestra los datos experimentales de las isotermas de adsorción de humedad obtenidas en equilibrio a $35^{\circ} \mathrm{C}$. Se observó una forma de tipo III (no sigmoidal) de acuerdo con Brunauer et al. (1940). Este comportamiento se debe al procesamiento dado, los cuales minimizan el área superficial del biopolímero al formar una película, disminuyendo su capacidad de adsorción de humedad (Gounavé et al., 2006).

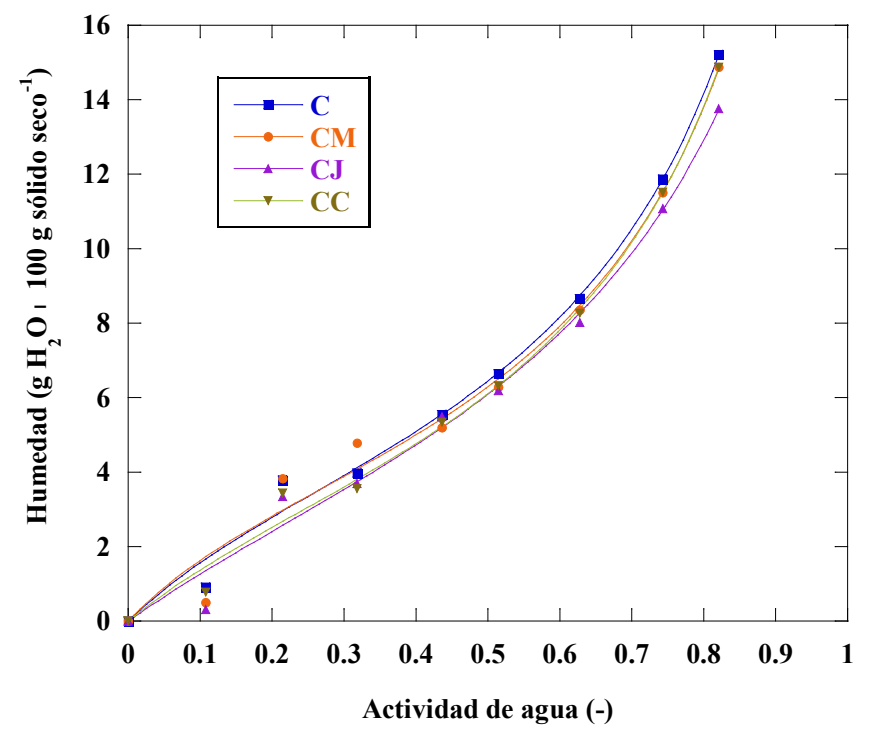

Figura 3. Isotermas de adsorción a $35^{\circ} \mathrm{C}$ de los bioplásticos. Control (C), cáscara de mango $(\mathrm{CM})$, cálices de jamaica cocidos $(\mathrm{CJ})$, residuos de café tostado (CC).

Figure 3. Bioplastics adsorption isotherms at $35^{\circ} \mathrm{C}$. Control (C) mango peel (CM), decocted roselle calyces (CJ), spent coffee grounds (CC).
La adsorción de vapor de agua en estos bioplásticos se atribuye a la formación de puentes de hidrógeno entre los grupos hidroxilo disponibles del almidón (Al-Muhtaseb et al., 2004), debido a que su carácter polar condiciona su capacidad de adsorción en la superficie. Se observó que formulaciones establecidas presentaron alta capacidad de adsorción de agua en valores de $\mathrm{a}_{\mathrm{w}}>0.3$ ( $4 \mathrm{~g}$ agua $/ 100 \mathrm{~g}$ sólido seco) y es similar a lo reportado por Peng et al. (2007), quienes evaluaron isotermas de adsorción del almidón de maíz en temperaturas similares. Este comportamiento se ve influenciado, entre otros factores, por el contenido de glicerol, debido a que su carácter hidrofílico incrementa las características higroscópicas de los bioplásticos (Mali et al., 2002; Bertuzzi et al., 2007). Sin embargo, se observó que los residuos adicionados no modificaron el tipo de las isotermas obtenidas.

Se determinaron los parámetros de la ecuación GAB (Mo, C, K) (Tabla 3). En este estudio, el modelo GAB se ajustó satisfactoriamente a los datos experimentales. El modelo de $G A B$ ha sido utilizado ampliamente para describir el comportamiento de las isotermas de adsorción de matrices alimentarias (Quirijns et al., 2005), ya que sus constantes tienen un significado físico y es capaz de ajustar los datos experimentales hasta una $a_{w}$ de 0.9 . Sin embargo, el valor de Mo obtenido del modelo GAB no es un indicador confiable de las condiciones óptimas de almacenamiento. En la mayoría de los casos, su aplicación debe ser corroborada experimentalmente (Maldonado et al., 2019). El valor de monocapa (Mo) es la cantidad de agua necesaria para formar una capa de moléculas que cubren la superficie del bioplástico. En la monocapa, la energía de interacción entre las moléculas de agua y la superficie del bioplástico no permite que el agua sea reactiva y participe en reacciones de deterioro. Las constantes $C$ y $\mathrm{K}$, proveen información acerca de la energía de interacción de las moléculas de agua en la monocapa y la multicapa, respectivamente (Quirijns et al., 2005). En la Tabla 3 , se muestra también el contenido de humedad correspondiente al volumen de microporo. Azuara y Beristain (2007) demostraron que en los microporos de matriz polimérica el agua interacciona con los sitios más activos de adsorción, formando una capa protectora contra la oxidación y a la vez, no participando como medio para reacciones de deterioro.

Se observaron diferencias significativas de los parámetros calculados entre los bioplásticos elaborados. Sin

Tabla 3. Parámetros de la ecuación de GAB y DR obtenidos a partir de las isotermas de adsorción de los bioplásticos a $35^{\circ} \mathrm{C}$. Table 3. GAB and DR equation parameters obtained from the bioplastics adsorption isotherms at $35^{\circ} \mathrm{C}$.

\begin{tabular}{lcccc}
\hline Parámetros & C & CM & CJ & CC \\
\hline Mo & $4.63 \pm 0.12 \mathrm{~b}$ & $4.30 \pm 0.01 \mathrm{c}$ & $5.24 \pm 0.11 \mathrm{a}$ & $4.52 \pm 0.07 \mathrm{~b}$ \\
$\mathrm{C}$ & $4.64 \pm 0.34 \mathrm{~b}$ & $5.37 \pm 0.67 \mathrm{a}$ & $3.20 \pm .22 \mathrm{~d}$ & $3.97 \pm .24 \mathrm{C}$ \\
$\mathrm{K}$ & $0.88 \pm 0.02 \mathrm{a}$ & $0.89 \pm 0.02 \mathrm{a}$ & $0.81 \pm 0.01 \mathrm{~b}$ & $0.88 \pm 0.02 \mathrm{a}$ \\
n0 & $5.05 \pm 0.03 \mathrm{a}$ & $4.98 \pm 0.12 \mathrm{ab}$ & $4.59 \pm 0.17 \mathrm{~b}$ & $4.62 \pm 0.34 \mathrm{~b}$ \\
$\mathrm{r}^{2}$ & 0.99 & 0.99 & 0.99 & 0.99 \\
\hline
\end{tabular}

Control (C), cáscara de mango (CM), cálices de jamaica cocidos (CJ), residuos de café tostado (CC). Letras distintas en la misma fila indican diferencias significativas $(\mathrm{p}<0.05)$ 
embargo, a pesar de las diferencias, la $\mathrm{a}_{\mathrm{w}}$ correspondiente a la monocapa de GAB y al volumen de microporo fue cercana a 0.4. La $a_{w}$ correspondiente a la monocapa de GAB para C, CM, CJ y CC fue de 0.36 (4.64 g agua/100 g sólido seco), 0.34 (4.30 agua/100 g sólido seco), 0.44 (5.24 agua/100 g sólido seco) y 0.38 (4.54 agua/100 g sólido seco), respectivamente. En el mismo sentido, la $a_{w}$ correspondiente al volumen de microporo para C, CM, CJ y CC fue de 0.39 (5.05 agua/100 g sólido seco), 0.39 (4.98 agua/100 g sólido seco), 0.38 (4.59 agua/100 g sólido seco) y 0.38 (4.62 agua/100 g sólido seco), respectivamente. Por lo tanto, se puede concluir que las condiciones óptimas de almacenamiento de los bioplásticos obtenidos a $35^{\circ} \mathrm{C}$, independientemente del subproducto agregado, es a una $a_{w}<0.4$. En esta $a_{w}$ el agua adsorbida no participará en reacciones de deterioro.

\section{Evaluación de las transiciones térmicas}

Los termogramas (Figura 4) muestran los cambios en la capacidad calorífica de los bioplásticos. Se encontró que $C$, $\mathrm{CM}, \mathrm{CJ}$ y $\mathrm{CC}$ exhibieron un evento endotérmico a una temperatura de $179^{\circ} \mathrm{C}, 178^{\circ} \mathrm{C}, 178^{\circ} \mathrm{C}$ y $172^{\circ} \mathrm{C}$, respectivamente, asociado a un proceso de fusión de las cucharas. En esta fase el material experimenta una transición de fase de sólido a líquido, lo que conlleva al ablandamiento del biopolímero (Worgull, 2009).

A temperaturas más elevadas, se observó un evento de oxidación en C, CJ y CC a una temperatura de $296^{\circ}$ C, mientras que en CM se presentó a $298^{\circ} \mathrm{C}$. La oxidación de las muestras se corresponde con un reordenamiento molecular y se observa como un fuerte aumento en el flujo de energía debido a la naturaleza exotérmica de las reacciones de oxidación. Este proceso se explica por el hecho de que la exposición prolongada de un material polimérico a una temperatura elevada propicia la escisión de las cadenas permitiendo al oxígeno atmosférico difundir a través de la superficie y reaccionar con la estructura del biopolímero (Kuakpetoon y Wang, 2008). Como consecuencia el almidón y la gelatina presentan una reorganización estructural; la oxidación introduce grupos carbonilo y carboxilo en las cadenas de carbonadas, dando lugar a un proceso inicial de degradación térmica. Estos resultados son congruentes con Beninca et al. (2008), quienes señalan que el tratamiento térmico del almidón conduce normalmente a su degradación cuando la temperatura aplicada supera los $300^{\circ} \mathrm{C}$. Un aumento adicional de la temperatura produce la carbonización del material polimérico, en donde las cadenas moleculares son destruidas por la energía térmica (Worgull, 2009).

A pesar de que se ha reportado que específicamente la adición de fibra a la matriz de almidón tiene un efecto favorecedor sobre la estabilidad térmica del biopolímero, aumentando la temperatura de degradación y disminuyendo la pérdida de masa (Xu y Hanna, 2009), en este estudio se observó que los eventos térmicos de todas las formulaciones fueron similares, por lo tanto, se puede establecer que
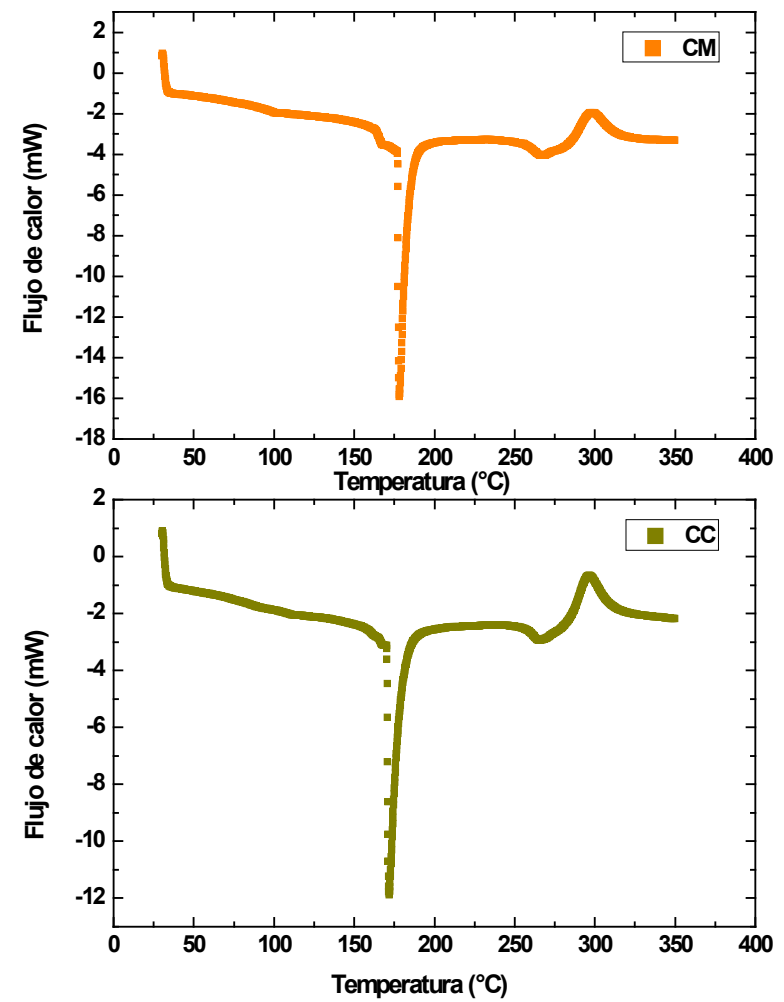

Figura 4. Termogramas DSC de los bioplásticos. Control (C), cáscara de mango (CM), cálices de jamaica cocidos (CJ), residuos de café tostado (CC).

Figure 4. Bioplastics DSC thermograms. Control (C) mango peel (CM), decocted roselle calyces (CJ), spent coffee grouds (CC). 
la adición de los subproductos agroindustriales a la matriz polimérica no afectó la estabilidad al calor de las cucharas. Este resultado también fue reportado por De Carvalho et al. (2018) al no observar cambios en las transiciones térmicas de películas de almidón adicionadas con fibras de bagazo de yuca. Los eventos térmicos de los biopolímeros fueron en general bastante uniformes, indicando una buena interacción entre los componentes glicerol, grenetina, almidón y fibras, por lo que no influyen en el mecanismo de descomposición de los bioplásticos, siendo esto favorable para la sustitución parcial de almidón y gelatina por subproductos sin un valor económico en la elaboración de estos.

\section{Biodegradación de los polímeros}

En la Figura 5 se muestra el aspecto físico de los biopolímeros a las 0,48 y 120 horas de su entierro en suelo con una $\mathrm{HR}=100 \%$. Durante el tiempo analizado, se observó un cambio de forma y un deterioro visual del color y de las propiedades mecánicas de los bioplásticos elaborados. A las $48 \mathrm{~h}$ del ensayo, independientemente del subproducto utilizado para la elaboración del bioplástico, se visualizó una notable pérdida de rigidez, dada por el agua absorbida del medio, la cual interacciona a nivel molecular con los componentes del biopolímero, favoreciendo su degradación (Hoover, 2001). La degradación de los biopolímeros que puede atribuirse a su composición. Diversos autores señalan que el proceso de desintegración se favorece en bioplásticos de almidón debido a que son fácilmente hidrolizados por amilasas y

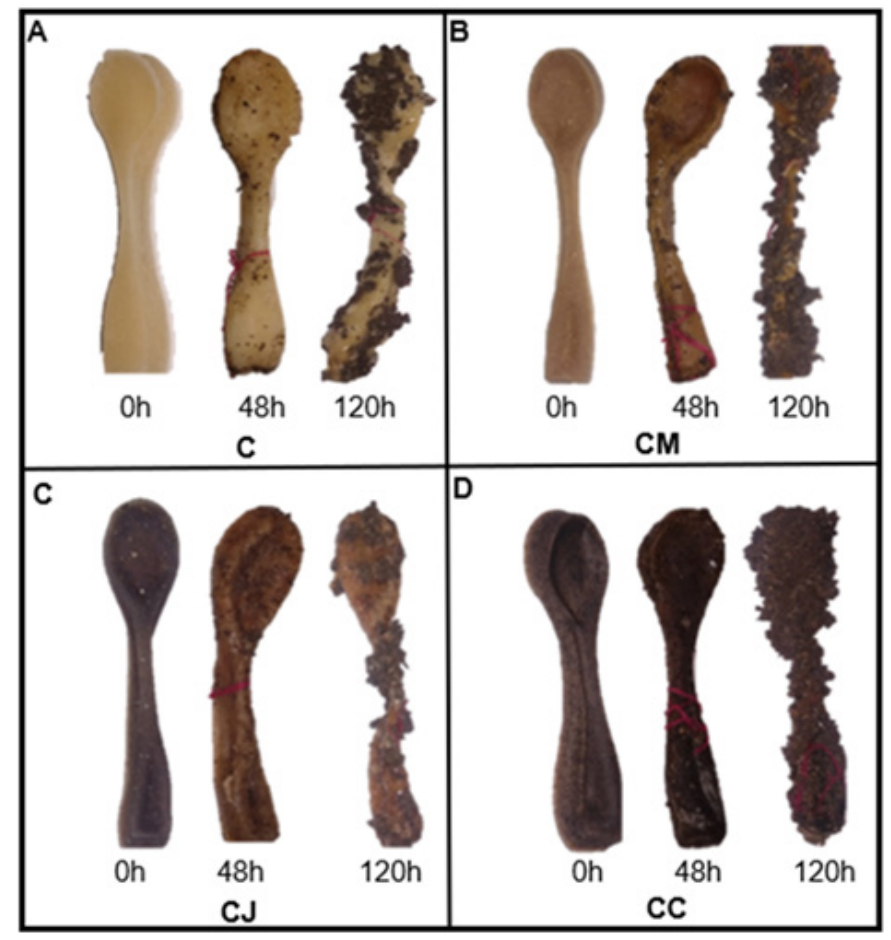

Figura 5. Prueba de degradación en suelo de los bioplásticos. A) Control (C); B) cáscara de mango (CM); C) cálices de jamaica cocidos (CJ); D) residuos de café tostado (CC).

Figure 5. Bioplastics soil burial test . A) Control (C); B) mango peel (CM); C) decocted roselle calyces (CJ); D) spent coffee grounds (CC). esterasas (Imam et al., 2000; Wang y Zhang, 2009). Después de $120 \mathrm{~h}$, independientemente del subproducto utilizado, la manipulación e identificación de los bioplásticos no fue posible debido a su fragmentación, hinchazón y pegajosidad. El tiempo de degradación observado en este estudio (5 días) fue menor al reportado por López-Velázquez et al. (2019) para hidrogeles de gelatina, quitosano, alcohol de polivinilo e inulina (28 días).

Estudios han reportado que la adición fibras naturales influye positivamente en la tasa de biodegradación debido a que actúan como canales que facilitan la entrada microbiana en la matriz polimérica. Franco et al. (2004) encontraron que la adición de fibras de agave en bioplásticos de almidónpolicaprolactona favoreció el ataque microbiano y la eliminación del almidón a través de la hidrólisis. Imam et al. (2005) observaron que la mezcla de almidón y la fibra de cascara de naranja con alcohol de polivinilo se degradaron en 30 días, mientras que la mezcla de almidón y alcohol de polivinilo en 120 días.

\section{CONCLUSIONES}

Este estudio exploró exitosamente el potencial de la adición de subproductos de mango, jamaica y café en la elaboración de un bioplástico de almidón-grenetina para elaborar cucharas biodegradables. La caracterización reveló que la adición de los subproductos agroindustriales modificó la capacidad de solubilización y aumentó la absorción de agua, así como la fuerza de fractura de los biopolímeros. Las condiciones óptimas de almacenamiento para los plásticos biodegradables fueron a actividades de agua inferiores a 0.4. Los bioplásticos exhibieron una adecuada estabilidad al calor (hasta $170^{\circ} \mathrm{C}$ ) y la adición de los subproductos no modificó las propiedades térmicas, siendo esto favorable para la sustitución parcial de almidón y gelatina por subproductos en la elaboración de estos. Los bioplásticos demostraron ser biodegradables en suelo. Por lo tanto, los subproductos de mango, jamaica y café, que actualmente no tienen un valor comercial, representan una fuente potencial de fibra que puede incorporarse a bioplásticos de almidón y gelatina para la obtención de cucharas biodegradables.

\section{REFERENCIAS}

Abarca, D., Martínez, R., Muñoz, J., Torres, M., y Vargas, G., 2010. Residuos de Café, Cacao y Cladodio de Tuna: Fuentes Promisorias de Fibra Dietaria. Revista Tecnológica-ESPOL, 23(2):63-69.

Al-Muhtaseb, A., Mcminn, W., y Magee, T. 2004. Water sorption isotherm of starch powders Part 1: Mathematical description of experimental data. Journal of Food Engineering. 61(3):297-307.

Aranda, F. J., González, R., Jasso, C. F., y Mendizábal, E. 2015. Water absorption and thermomechanical characterization of extruded starch/poly (lactic acid)/agave bagasse fiber bioplastic composites. International Journal of Polymer Science, 2015: 1-7.

Ashori, A., y Nourbakhsh, A. 2010. Bio-based composites from waste agricultural residues. Waste Management, 30(4): 680684. 
ASTM. 2005. Standard test method for plastics. In: Annual Book of ASTM Standards. American Society for Testing and Materials, Philadelphia, PA.

Avérous, L. y Boquillon, N. 2004. Biocomposites based on plasticized starch: thermal and mechanical behaviors. Carbohydrate Polymer. 56:111-122.

Azuara-Nieto, E., y Beristain-Guevara, C. I. (2007). Estudio termodinámico y cinético de la adsorción de agua en proteína de suero de leche. Revista Mexicana de Ingeniería Química, 6(3), 359-365.

Beninca, C., Demiate, I. M., Lacerda, L. G., Carvalho Filho, M. A. D. S., Ionashiro, M., y Schnitzler, E. 2008. Thermal behavior of corn starch granules modified by acid treatment at 30 and 50 C. Eclética Química, 33(3), 13-18.

Bertuzzi, M. A., Armada, M., y Gottifredi, J. C. 2007. Physicochemical characterization of starch-based films. Journal of Food Engineering, 82(1): 17-25.

Brizga, J., Hubacek, K., y Feng, K. (2020). The Unintended Side Effects of Bioplastics: Carbon, Land, and Water Footprints. One Earth, 3(1), 45-53.

Brunauer, S., Deming, L. S., Deming, W. E., y Teller, E. 1940. On a theory of the van der Waals adsorption of gases. Journal of the American Chemical society, 62(7), 1723-1732.

Curvelo, A., De Carvalho, A., Agnelli, J. 2001. Thermoplastic starch-cellulosic fibers composites: Preliminary results. Carbohydrate Polymers. 45:183-188.

De Carvalho, G. R., Marques, G. S., de Matos Jorge, L. M., y Jorge, R. M. M. (2018). Cassava bagasse as a reinforcement agent in the polymeric blend of biodegradable films. Journal of Applied Polymer Science, 47224.

Franco, C., Cyras, V., Busalmen, J., Ruseckaite, R. y Vázquez, A. 2004. Degradation of polycaprolactone/starch blends and composites with sisal fibre. Polymer Degradation and Stability, 86:95-103.

García-Gurrola, A., Rincón, S., Escobar-Puentes, A. A., Zepeda, A., y Martínez-Bustos, F. 2019. Microencapsulation of red sorghum phenolic compounds with esterified sorghum starch as encapsulant materials by spray drying. Food Technology and Biotechnology, 57(3), 341.

Gáspar, M., Benkó, Z., Dogossy, G., Réczey, K., y Czigány, T. 2005. Reducing water absorption in compostable starch-based plastics. Polymer Degradation and Stability. 90:563-569.

Gounavé, F., Marais, S., Bessadok, A., Lan Gevin, D., Morvan, C., y Métayer, M. 2006. Study of water sorption in modified flax fibers. Journal of Applied Polymer Science. 101:4281-4289.

Hoover, R. 2001. Composition, molecular structure, and physicochemical properties of tuber and root starches: a review. Carbohydrate Polymer. 45:253-267.

Imam, S. H., Cinelli, P., Gordon, S. H., y Chiellini, E. 2005. Characterization of biodegradable composite films prepared from blends of poly (vinyl alcohol), cornstarch, and lignocellulosic fiber. Journal of Polymers and the Environment. 13(1):47-55.

Imam, S., Gordon, S., Shogren, R., Tosteson, T., Govind, N. y Greene, R. 2000. Degradation of starch-poly(b-hydroxybutyrateco-b-hydroxvalerate) bioplastic in tropical coastal waters. Applied and Environmental Microbiology. 65:431-437.

Jumaidin, R., Khiruddin, M. A. A., Saidi, Z. A. S., Salit, M. S., y Ilyas, R. A. (2020). Effect of cogon grass fibre on the thermal, mechanical and biodegradation properties of thermoplastic cassava starch biocomposite. International Journal of Biological Macromolecules, 146, 746-755.
Kaisangsri, N., Kerdchoechuen, O., y Laohakunjit, N. (2014). Characterization of cassava starch based foam blended with plant proteins, kraft fiber, and palm oil. Carbohydrate Polymers, 110, 70-77.

Krishnamurthy, A., y Amritkumar, P. (2019). Synthesis and characterization of eco-friendly bioplastic from low-cost plant resources. SN Applied Sciences, 1(11), 1432.

Kuakpetoon, D., y Wang. Y. 2008. Locations of hypochlorite oxidation in corn starches varying in amylose content. Carbohydrate Research. 343:90-100.

López-Velázquez, J. C., Rodríguez-Rodríguez, R., EspinosaAndrews, H., Qui-Zapata, J. A., García-Morales, S., NavarroLópez, D. E., y Luna-Barcénas, G., Vassallo-Brigneti, E. T. y García-Carvajal, Z. Y. (2019). Gelatin-chitosan-PVA hydrogels and their application in agriculture. Journal of Chemical Technology \& Biotechnology, 94(11), 3495-3504.

Lubis, M., Gana, A., Maysarah, S., Ginting, M. H. S., y Harahap, M. B. 2018. Production of bioplastic from jackfruit seed starch (Artocarpus heterophyllus) reinforced with microcrystalline cellulose from cocoa pod husk (Theobroma cacao L.) using glycerol as plasticizer. IOP Conference Series: Materials Science and Engineering. 309:012100.

Lucas, N., Bienaime, C. and Belloy, C. 2008. Polymer biodegradation: Mechanisms and estimation techniques - $\mathrm{A}$ review, Chemosphere. 73(4):429-442.

McNutt, J. (2019). Spent coffee grounds: A review on current utilization. Journal of industrial and engineering chemistry, 71, 78-88.

Maldonado, Y., Jiménez, J., Arámbula, G., Flores, V., Álvarez, P., Ramírez, M., y Salazar, R. 2019. Effect of water activity on extractable polyphenols and some physical properties of Hibiscus sabdariffa L. calyces. Journal of Food Measurement and Characterization. 13(1):687-696.

Mali, S., Grossmann, M. V. E., Garcia, M. A., Martino, M. N., y Zaritzky, N. E. (2002). Microstructural characterization of yam starch films. Carbohydrate Polymers, 50(4), 379-386.

Mayo-Mayo, G., Navarrete-García, A., Maldonado-Astudillo, Y. I., Jiménez-Hernández, J., Santiago-Ramos, D., ArámbulaVilla, G., Álvarez-Fitz, P., Ramírez, M. y Salazar, R. (2020). Addition of roselle and mango peel powder in tortilla chips: a strategy for increasing their functionality. Journal of Food Measurement and Characterization, 14, 1511-1519.

Navia, D., Ayala, A. y Villada, H. 2011. Adsorption isotherms of cassava flour bioplastics compression molded. Biotecnología en el Sector Agropecuario y Agroindustrial. 9(1):77-87.

Moro, T. M., Ascheri, J. L., Ortiz, J. A., Carvalho, C. W., y MeléndezArévalo, A. (2017). Bioplastics of native starches reinforced with passion fruit peel. Food and Bioprocess Technology, 10(10), 1798-1808.

Peng, G., Chen, X., Wu, W., y Jiang, X. 2007. Modeling of water sorption isotherm for corn starch. Journal of Food Engineering, 80(2):562-567.

Quirijns, E. J., van Boxtel, A. J., van Loon, W. K., y van Straten, G. 2005. Sorption isotherms, GAB parameters and isosteric heat of sorption. Journal of the Science of Food and Agriculture. 85(11):1805-1814.

Rodríguez, W., Flores, J., Martınez, F., Chinas, F., Espinoza, F. 2014. Nanomechanical properties and thermal stability of recycled cellulose reinforced starch-gelatin polymer composite. The Journal of Applied Polymer Science. 132(4). 
Santillán-Moreno, A., Martínez-Bustos, F., Castaño-Tostado, E., y Amaya-Llano, S. L. (2011). Physicochemical characterization of extruded blends of corn starch-whey protein concentrate-Agave tequilana fiber. Food and Bioprocess Technology, 4(5), 797-808.

Syamani, F. A., Pramasari, D. J., Kusumaningrum, W. B., Kusumah, S. S., Masruchin, N., Ermawati, R., y Cahyaningtyas, A. A. (2020). Characteristics of Bioplastic Made from Cassava Starch Filled with Fibers from Oil Palm Trunk at Various Amount. E\&ES, 439(1), 012035.

Torres, F. G., Mayorga, J. P., Vilca, C., Arroyo, J., Castro, P., y Rodriguez, L. (2019). Preparation and characterization of a novel starch-chestnut husk biocomposite. SN Applied Sciences, 1(10), 1158.

Väisänen, T., Haapala, A., Lappalainen, R., y Tomppo, L. 2016. Utilization of agricultural and forest industry waste and residues in natural fiber-polymer composites: A review. Waste Management. 54:62-73.

Velásquez, S., Pelaéz, G., Giraldo, D. 2016. Use of vegetable fibers in polymer matrix composites: a review with a view to their application in designing new products. Informador Técnico (Colombia). 80(1):77-86.
Williamson, K., y Hatzakis, E. (2019). NMR analysis of roasted coffee lipids and development of a spent ground coffee application for the production of bioplastic precursors. Food Research International, 119, 683-692.

Worgull, M. 2009. Molding Materials for Hot Embossing. En: Hot Embossing: Theory and Technology of Microreplication. William Andrew (ed.), pp 57-112, Oxford, UK.

Xu, Y. y Hanna, M. 2005. Preparation and properties of biodegradable foams from starch acetate and poly(tetramethylene adipate-co-terephthalate). Carbohydrate Polymer. 59:521-529.

Yang, J., Ching, Y. C., y Chuah, C. H. (2019). Applications of lignocellulosic fibers and lignin in bioplastics: A review. Polymers, 11(5), 751.

Wang, Y., y Zhang, L. (2009). In L. Yu (Ed.), Biodegradable polymer blends and composites from renewable resources (pp. 129161). New Jersey: Wiley. Part I, Chapter 6.

Wang, W., Wang, H., Jin, X., Wang, H., Lin, T., y Zhu, Z. (2018). Effects of hydrogen bonding on starch granule dissolution, spinnability of starch solution, and properties of electrospun starch fibers. Polymer, 153, 643-652. 